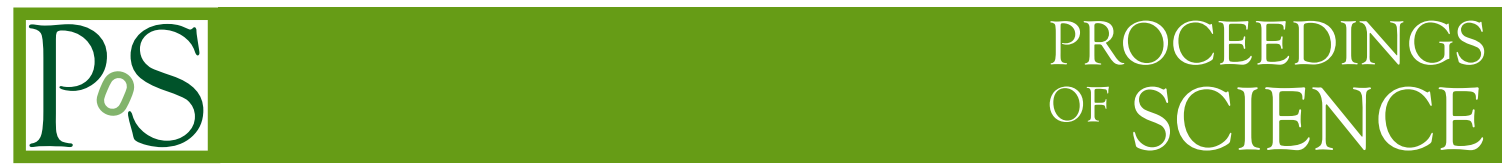

\title{
Leptonic Scalars versus Scalar Leptons
}

\author{
Ernest Ma*† \\ Department of Physics and Astronomy, University of California, Riverside \\ E-mail: ma@phyun8.ucr.edu
}

The notion of scalars having lepton number, i.e. leptonic scalars, is discussed without supersymmetry, where scalar leptons reside. It is shown how dark parity comes from lepton parity, as well as its possible origin from grand unification. Two specific phenomenological applications to dark matter are described.

Corfu Summer Institute 2019 "School and Workshops on Elementary Particle Physics and Gravity" (CORFU2019)

31 August - 25 September 2019

Corfu, Greece

\footnotetext{
*Speaker.

${ }^{\dagger}$ Supported in part by the U.S. Department of Energy under Grant No. DE-SC0008541.
} 


\section{The SusyVerse}

In the Standard Model (SM), there are fermions which have either baryon number $B=1 / 3$ (quarks) or lepton number $L=1$ (leptons), and vector gauge bosons $B=L=0$, and Higgs bosons $B=L=0$. In its supersymmetric (susy) extension, the scalar partners of the fermionic quarks and leptons are then naturally known as scalar quarks and scalar leptons. Since they share the same $B$ and $L$, what distinguishes them is spin, i.e. $R$ parity, defined as $(-1)^{3 B+L+2 j}$.

It is well-known that $B$ and $L$ are automatic symmetries in the SM. If neutrinos are Majorana, then $L$ becomes lepton parity $(-1)^{L}$. In susy, $B$ and $(-1)^{L}$ are not automatic, but if they are assumed to be conserved, then odd $R$ parity defines the dark sector, the lightest particle of which is assumed to be neutral and becomes a candidate for the dark matter of the Universe. This notion is one of the main reasons of the push to discover the SusyVerse in the past four decades. Note that scalar leptons are always connected to leptons through gauginos and higgsinos in susy.

In general, once new particles are added, their $B$ and $L$ assignments are not automatic, but dictated by how they interact with the SM particles. This is especially true of a neutral fermion singlet. It does NOT have to be a right-handed neutrino, as most people would assume without thinking twice. See my Brief Review [1] of 2017. In this talk, I will touch upon some other recent new ideas in extending $L$ to accommodate the existence of dark matter (DM).

\section{Dark Parity from Lepton Parity}

Prototype models of dark matter invariably began with the assumption of a dark $Z_{2}$ symmetry, under which the SM particles are even and those of the dark sector are odd. However, this has been shown [2] to be unnecessary because dark parity is easily derived from lepton parity. Start with the SM with no susy, add a real singlet scalar $S$ for DM [3]. The usual and obvious assumption is to postulate a new $Z_{2}$ symmetry as mentioned above. However, the same Lagrangian is obtained if lepton parity is used, under which all known leptons are odd as well as $S$. Now the latter, being a scalar, has odd dark parity, and may be called a leptonic scalar. It is not a scalar lepton in the sense of supersymmetry.

Another minimal addition [4] to the SM is that of a real singlet scalar $S$ and a singlet Majorana fermion $\chi_{L}$ in the presence of a singlet right-handed neutrino $N_{R}$. The conventional assumption for dark matter is again $Z_{2}$ under which $S$ and $\chi_{L}$ are odd, and all SM particles are even including $N_{R}$. This allows the Yukawa interaction $\bar{\chi}_{L} N_{R} S$ and either $\chi_{L}$ or $S$ could be DM. Once more, the dark $Z_{2}$ is not necessary; the same Lagrangian is obtained if $S$ has odd, and $\chi_{L}$ has even lepton parity, so that they both have odd dark parity.

The notion of using lepton parity assignments to new additional scalars and fermions to the SM is applicable also to all generic models of radiative Majorana neutrino mass through DM, i.e. the scotogenic mechanism. For example, in the well-known one-loop model [5], instead of the original assumption that the second scalar doublet $\left(\eta^{+}, \eta^{0}\right)$ and the singlet Majorana fermions $N$ be odd under a new dark $Z_{2}$, they may simply be assigned odd and even lepton parity. Hence $\left(\eta^{+}, \eta^{0}\right)$ is a leptonic scalar doublet, and not a scalar lepton doublet. 


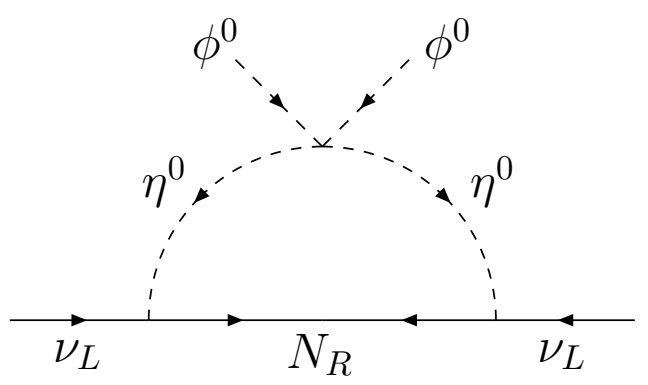

Figure 1: Radiative seesaw neutrino mass: the scotogenic mechanism.

\section{GUT Origin of Dark Parity}

The conventional definition of $R$ parity may be written for SM particles as $(-1)^{3(B-L)+2 j}[6]$. This suggests strongly its origin from $S O(10) \rightarrow S U(3)_{C} \times S U(2)_{L} \times S U(2)_{R} \times U(1)_{B-L}$. In the $\underline{16}$ repreentation of $S O(10)$, quarks and their conjugates have $B-L= \pm 1 / 3$, whereas leptons and their conjugates have $B-L=\mp 1$. In the 10 representation, the color triplets have $B-L=\mp 2 / 3$ and the left-right bidoublet has $B-L=0$. Hence $(-1)^{3(B-L)}$ is odd and even respectively. This means that $R$ parity is even/odd and odd/even for fermion/scalar in the $\underline{16}$ and $\underline{10}$ representations respectively. Hence $B-L$ is a possible marker symmetry for dark matter.

However, $U(1)_{B-L}$ is not orthogonal to $U(1)_{Y}$ of the $\mathrm{SM}$, and since a dark-matter candidate is likely to be a trivial singlet under $S U(3)_{C} \times S U(2)_{L} \times U(1)_{Y}$, a better choice is $U(1)_{\chi}$ from the decomposition $S O(10) \rightarrow S U(5) \times U(1)_{\chi}$ [7]. Now $\underline{16}=\left(5^{*}, 3\right)+(10,-1)+(1,-5), \underline{10}=$ $\left(5^{*},-2\right)+(5,2)$. Using $3 Q_{\chi}=12 Y-15(B-L)$, a good marker symmetry for dark matter is $R_{\chi}=(-1)^{Q_{\chi}+2 j}$. The so-called right-handed neutrino is now a singlet, denoted by its conjugate $v^{c} \sim(1,-5)$, instead of belonging to an $S U(2)_{R}$ doublet. In this context, previous dark-matter assignments are $S \sim(1,-5), \chi \sim(1,0),\left(\eta^{+}, \eta^{0}\right) \sim(5,-3)$, and $N \sim(1,0)$. Gauge $U(1)_{\chi}$ is broken by a scalar $(1,1,0,-10)$ resulting in $Z_{\chi}$ with $m_{Z_{\chi}}>4.1 \mathrm{TeV}$ from LHC data. Interestingly, the addition of DM scalar $(1,3,0,-5)$ and fermions $(1,3,0,0),(8,1,0,0)$ allows gauge coupling unification without supersymmetry.

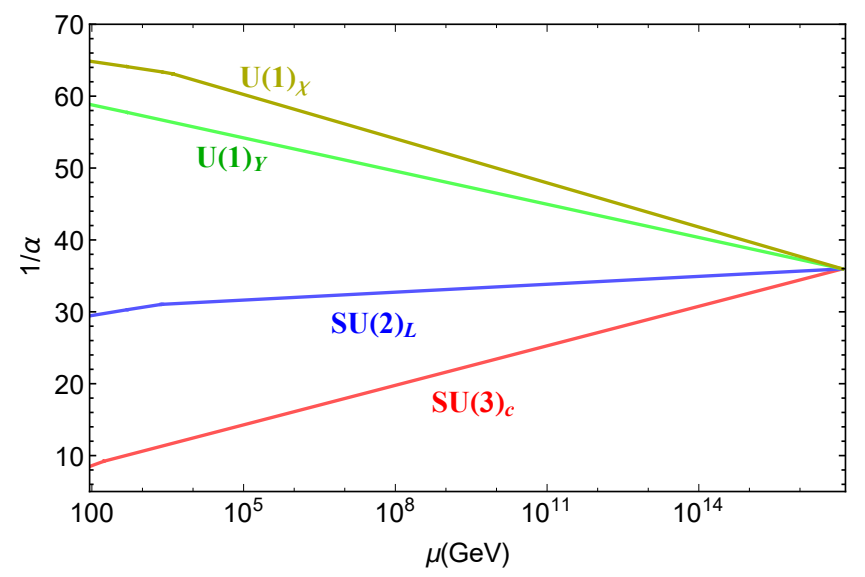

Figure 2: Running of $1 / \alpha_{i}$ with energy scale. 


\section{SIDM with Leptonic and Dileptonic Scalars}

Lepton number is usually thought of as being an integer $L$ or a parity $(-1)^{L}$. In the latter case, neutrinos are Majorana, which is the default option. In the former case, they are Dirac, and in the persisting nonobservation of neutrinoless double beta decay, there is a theoretical resurgence of interest in them. In particular, leptonic and dileptonic scalars may be postulated for dark matter and its light mediator in a simple model [8] of self-interacting dark matter (SIDM) to solve the cusp-core discrepancy in the profile of dwarf galaxies. Under $L$, let the complex scalars $\chi \sim 1$, $\zeta \sim 2$, implying thus allowed cubic $\mu_{12} \zeta^{*} \chi^{2}$ and quartic $\lambda_{12}\left(\chi^{*} \chi\right)\left(\zeta^{*} \zeta\right)$ interactions. The elastic scattering of $\chi$ through $\zeta$ exchange has the cross section

$$
\sigma_{e l}\left(\chi \chi^{*} \rightarrow \chi^{*} \chi\right)=\frac{\mu_{12}^{4}}{4 \pi m_{\chi}^{2} m_{\zeta}^{4}} .
$$

Its annihilation to $\zeta$ has the cross section

$$
\sigma_{a n n}\left(\chi \chi^{*} \rightarrow \zeta \zeta^{*}\right) v_{r e l}=\frac{1}{32 \pi m_{\chi}^{2}}\left(\lambda_{12}-\frac{2 \mu_{12}^{2}}{m_{\chi}^{2}}\right)^{2}
$$

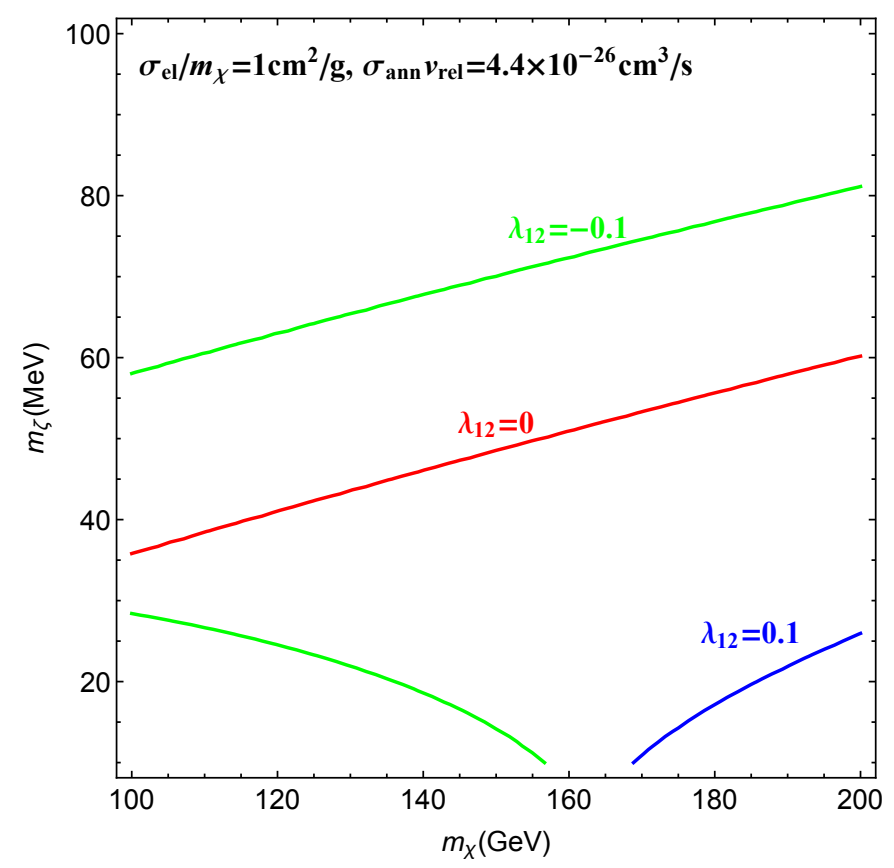

Figure 3: Parameter space of $m_{\zeta}$ versus $m_{\chi}$.

Using the benchmark values $\sigma_{e l}=1 \mathrm{~cm}^{2} / \mathrm{g}$ and $\sigma_{a n n} v_{r e l}=4.4 \times 10^{-26} \mathrm{~cm}^{3} / \mathrm{s}$, the masses of $\zeta$ and $\chi$ are shown to be in the range 10 to $80 \mathrm{MeV}$ and 100 to $200 \mathrm{GeV}$ respectively. What distinguishes this application of leptonic scalar to SIDM is that the light mediator has $L=2$. In the conventional scenario, this mediator is either a light gauge boson (which mixes kinetically with the photon) or a light scalar (which mixes with the SM Higgs boson). In either case, it will decay to electrons and would disrupt the CMB (Cosmic Microwave Background) when its production gets enhanced by the Sommerfeld effect in late times $[9,10]$. Here, $\zeta$ decays only to two neutrinos!! 


\section{Radiative Dileptonic Dark Fermion Mass}

A new application of lepton number for dark matter is a variation of the $S / \chi$ model. Under $L$, let $\zeta \sim 1$ and $\chi_{L} \sim 2$ in the presence of $N_{R}$. All dimension- 4 terms of the Lagrangian including $f \bar{\chi}_{L} N_{R} \zeta$ obey $L$, whereas the dimension-2 term $\mu^{2}\left[\zeta^{2}+\left(\zeta^{*}\right)^{2}\right] / 2$ and the dimension-3 term $\left(m_{N} / 2\right) N_{R} N_{R}+$ H.c. break it softly by 2 units. Here neutrinos obtain Majorana masses through the conventional seesaw mechanism, i.e. $m_{v} \simeq m_{D}^{2} / m_{N}$, while the dark fermion $\chi$ obtains a radiative mass in one loop, also anchored by $m_{N}$.

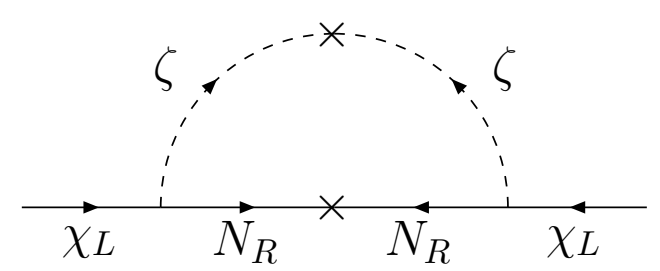

Figure 4: Radiative seesaw mass for $\chi$.

Let $\zeta=\left(\zeta_{R}+i \zeta_{I}\right) / \sqrt{2}$ with masses $m_{R}, m_{I}$, split by the $\mu^{2}$ term, then

$$
m_{\chi}=\frac{f^{2} m_{N}}{32 \pi^{2}}\left[\frac{m_{N}^{2}}{m_{R}^{2}-m_{N}^{2}} \ln \frac{m_{R}^{2}}{m_{N}^{2}}-\frac{m_{N}^{2}}{m_{I}^{2}-m_{N}^{2}} \ln \frac{m_{I}^{2}}{m_{N}^{2}}\right]
$$

is the analog of the scotogenic neutrino mass. For $m_{R}^{2}-m_{I}^{2}=\mu^{2}<<m_{0}^{2}=\left(m_{R}^{2}+m_{I}^{2}\right) / 2<<m_{N}^{2}$,

$$
m_{\chi}=\frac{f^{2} \mu^{2}}{16 \pi^{2} m_{N}}\left[\ln \frac{m_{N}^{2}}{m_{N}^{2}}-1\right]
$$

Let $m_{N}=10^{6} \mathrm{GeV}, m_{0}=1 \mathrm{TeV}, \mu=100 \mathrm{GeV}, f=0.35$, then $m_{\chi}=0.1 \mathrm{MeV}$; whereas $m_{D}=10$ $\mathrm{MeV}$ yields $m_{v}=01 \mathrm{eV}$.

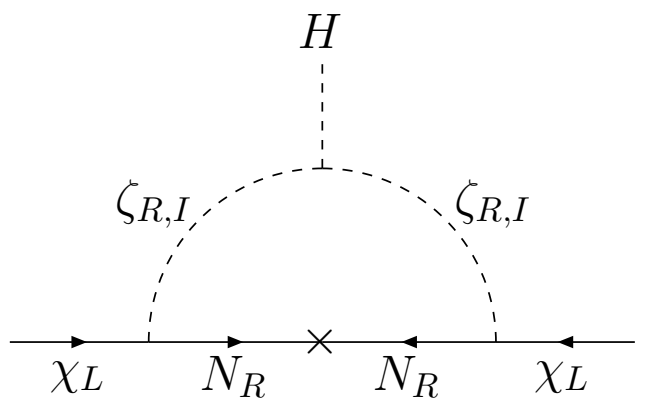

Figure 5: One-loop effective Higgs coupling to $\chi \chi$.

The dark fermion $\chi$ interacts only through the very heavy $\zeta$ and $N$ particles. If the reheat temperature of the Universe is much below $m_{N}, \chi$ may be produced only by the freeze-in mechanism through Higgs decay. The effective one-loop coupling $f_{H}$ of $H$ to $\chi \chi$ is

$$
\frac{\lambda_{3} v f^{2} m_{N}}{32 \pi^{2}}\left[\frac{1}{m_{R}^{2}-m_{N}^{2}}-\frac{m_{N}^{2} \ln \left(m_{R}^{2} / m_{N}^{2}\right)}{\left(m_{R}^{2}-m_{N}^{2}\right)^{2}}-\left(m_{R}^{2} \rightarrow m_{I}^{2}\right)\right]
$$


where $\lambda_{3}$ is the $\left(\Phi^{\dagger} \Phi\right)\left(\zeta^{*} \zeta\right)$ coupling and $v / \sqrt{2}$ the vacuum expectation value of $\phi^{0}$. It is proportional to $m_{\chi}$ with the factor $\lambda_{3} v / m_{0}^{2}\left[\ln \left(m_{N}^{2} / m_{0}^{2}\right)-1\right]$. The decay rate of the SM Higgs boson to $\chi \chi$ is

$$
\Gamma_{H}=\frac{f_{H}^{2} m_{H}}{8 \pi} \sqrt{1-4 x^{2}}\left(1-2 x^{2}\right),
$$

where $x=m_{\chi} / m_{H}$. The production of $\chi$ is through $H$ decay before the latter decouples from the thermal bath. For $x<<1$, the correct relic abundance from freeze-in through Higgs decay is obtained for $f_{H} \sim 10^{-12} x^{-1 / 2}$. In this example, it is satisfied for $\lambda_{3}=0.58$. Thus $\chi$ is a possible feebly interacting light dark fermion.

\section{Concluding Remarks}

Lepton parity and lepton number are useful concepts for extending the non-susy SM to include dark matter. In the grand-unified theory context, dark parity may be derived from $Q_{\chi}$ based on $S O(10) \rightarrow S U(5) \times U(1)_{\chi}$. Using exact $L$ with Dirac neutrinos, self-interacting dark matter is possible where the light mediator decays only to two neutrinos, thereby not disrupting the CMB. Using softly broken $L$ with Majorana neutrinos, a drak fermion may acquire a small radiative Majorana mass and becomes freeze-in dark matter through Higgs decay.

\section{References}

[1] E. Ma, Mod. Phys. Lett. A32, 1730007 (2017).

[2] E. Ma, Phys. Rev. Lett. 115, 011801 (2015).

[3] V. Silveira and A. Zee, Phys. Lett. 161B, 136 (1985).

[4] M. Pospelov, A. Ritz, and M. B. Voloshin, Phys. Lett. B662, 53 (2008).

[5] E. Ma, Phys. Rev. D73, 077301 (2006).

[6] S. P. Martin, Phys. Rev. D46, R2769 (1992).

[7] E. Ma, Phys. Rev. D98, 091701(R) (2018).

[8] E. Ma, Mod. Phys. Lett. A33, 1850226 (2018).

[9] S. Galli, F. Iocco, G. Bertone, and A. Melchiorri, Phys. Rev. D80, 023505 (2009).

[10] T. Bringmann, F. Kahlhoefer, K. Schmidt-Hoberg, and P. Walia, Phys. Rev. Lett. 118, 141802 (2017). 